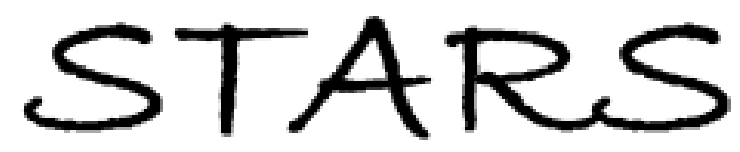

University of Central Florida

STARS

$1-1-2009$

\title{
Magnetic Field Topology in Low-Mass Stars: Spectropolarimetric Observations of M Dwarfs
}

Ngoc Phan-Bao

Jeremy Lim

Jean-François Donati

Christopher M. Johns-Krull

Eduardo L. Martín

University of Central Florida

Find similar works at: https://stars.library.ucf.edu/facultybib2000

University of Central Florida Libraries http://library.ucf.edu

This Article is brought to you for free and open access by the Faculty Bibliography at STARS. It has been accepted for inclusion in Faculty Bibliography 2000s by an authorized administrator of STARS. For more information, please contactSTARS@ucf.edu.

\section{Recommended Citation}

Phan-Bao, Ngoc; Lim, Jeremy; Donati, Jean-François; Johns-Krull, Christopher M.; and Martín, Eduardo L., "Magnetic Field Topology in Low-Mass Stars: Spectropolarimetric Observations of M Dwarfs" (2009).

Faculty Bibliography 2000s. 1952.

https://stars.library.ucf.edu/facultybib2000/1952

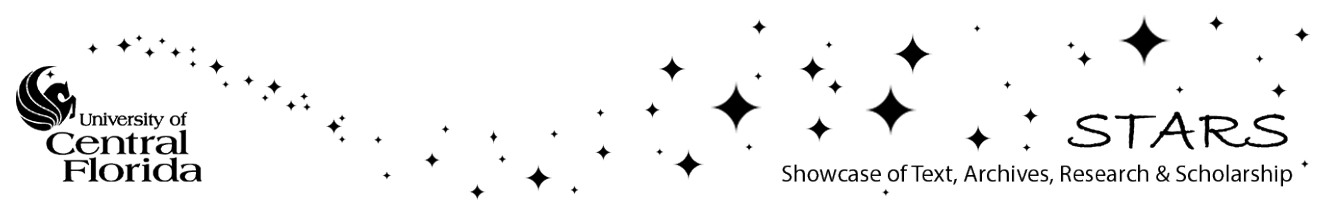




\title{
MAGNETIC FIELD TOPOLOGY IN LOW-MASS STARS: SPECTROPOLARIMETRIC OBSERVATIONS OF M DWARFS*
}

\author{
Ngoc Phan-Bao ${ }^{1,2}$, Jeremy Lim ${ }^{1,3}$, Jean-François Donati ${ }^{4}$, Christopher M. Johns-Krull ${ }^{5}$, and Eduardo L. Martín ${ }^{6,7}$ \\ ${ }^{1}$ Institute of Astronomy and Astrophysics, Academia Sinica, P.O. Box 23-141, Taipei 106, Taiwan; pbngoc@asiaa.sinica.edu.tw, jlim@asiaa.sinica.edu.tw \\ ${ }^{2}$ Department of Physics, HCMIU, Vietnam National University Administrative Building, Block 6, Linh Trung Ward, Thu Duc District, HCM, Vietnam \\ ${ }^{3}$ Department of Physics, Room 518, Chong Yuet Ming Physics Building, The University of Hong Kong, Pokfulam Road, Hong Kong, China \\ ${ }^{4}$ Laboratoire d'Astrophysique, Observatoire Midi-Pyrénées, F-31400 Toulouse, France; donati@ast.obs-mip.fr \\ ${ }^{5}$ Department of Physics and Astronomy, Rice University, 6100 Main Street, MS-61 Houston, TX 77005, USA; cmj@rice.edu \\ ${ }^{6}$ CSIC-INTA Centro de Astrobiologia, Torrejon de Ardoz, Madrid, Spain; ege@iac.es \\ ${ }^{7}$ University of Central Florida, Department of Physics, P.O. Box 162385, Orlando, FL 32816-2385, USA \\ Received 2009 June 30; accepted 2009 September 14; published 2009 October 6
}

\begin{abstract}
The magnetic field topology plays an important role in the understanding of stellar magnetic activity. While it is widely accepted that the dynamo action present in low-mass partially convective stars (e.g., the Sun) results in predominantly toroidal magnetic flux, the field topology in fully convective stars (masses below $\sim 0.35 M_{\odot}$ ) is still under debate. We report here our mapping of the magnetic field topology of the M4 dwarf G 164-31 (or Gl 490B), which is expected to be fully convective, based on time series data collected from $20 \mathrm{hr}$ of observations spread over three successive nights with the ESPaDOnS spectropolarimeter. Our tomographic imaging technique applied to time series of rotationally modulated circularly polarized profiles reveals an axisymmetric large-scale poloidal magnetic field on the M4 dwarf. We then apply a synthetic spectrum fitting technique for measuring the average magnetic flux on the star. The flux measured in $\mathrm{G} \mathrm{164-31}$ is $|B f|=3.2 \pm 0.4 \mathrm{kG}$, which is significantly greater than the average value of $0.68 \mathrm{kG}$ determined from the imaging technique. The difference indicates that a significant fraction of the stellar magnetic energy is stored in small-scale structures at the surface of $\mathrm{G}$ 164-31. Our $\mathrm{H} \alpha$ emission light curve shows evidence for rotational modulation suggesting the presence of localized structure in the chromosphere of this $\mathrm{M}$ dwarf. The radius of the M4 dwarf derived from the rotational period and the projected equatorial velocity is at least $30 \%$ larger than that predicted from theoretical models. We argue that this discrepancy is likely primarily due to the young nature of G 164-31 rather than primarily due to magnetic field effects, indicating that age is an important factor which should be considered in the interpretation of this observational result. We also report here our polarimetric observations of five other M dwarfs with spectral types from M0 to M4.5, three of them showing strong Zeeman signatures.
\end{abstract}

Key words: stars: individual (G 164-31, Gl 890, LHS 473, KP Tau, Gl 896B, 2E 4498) - stars: low-mass, brown dwarfs - stars: magnetic fields - techniques: polarimetric - techniques: spectroscopic

Online-only material: color figures

\section{INTRODUCTION}

Magnetic fields in the Sun and partially convective stars (e.g., G, K stars and possibly early-M dwarfs) are produced by the so-called $\alpha \Omega$ dynamo mechanism operating at the interface between the convective envelope and the radiative core, where differential rotation is strongest, known as the tachocline. The action of differential rotation on a weak poloidal field at the base of the convective zone results in a large-scale and predominantly toroidal subsurface field produced by this dynamo action (Parker 1975). However, when this toroidal field emerges through the surface of the Sun, the magnetic flux tubes are oriented in a primarily radial direction in the photosphere (e.g., see the review by Solanki 2009).

Stars with masses below $\sim 0.35 M_{\odot}(\sim \mathrm{M} 3)$ are expected to be fully convective as suggested by standard models (e.g., Chabrier \& Baraffe 1997). This limit probably shifts toward lower masses due to the influence of the magnetic field (Mullan $\&$ MacDonald 2001; Chabrier et al. 2007). In fully convective stars, the lack of a radiative core is expected to preclude the

\footnotetext{
* Based on observations made at the Canada-France-Hawaii Telescope, operated by the National Research Council of Canada, the Centre National de la Recherche Scientifique de France and the University of Hawaii.
}

$\alpha \Omega$ dynamo, maintained by the combined action of differential rotation $(\Omega$ effect) and cyclonic convection ( $\alpha$ effect). This raises the question of what type of dynamo produces strong magnetic activity as observed in mid- to late-M and brown dwarfs from photospheric Zeeman splitting (Johns-Krull \& Valenti 1996; Reiners \& Basri 2009) to chromospheric $\mathrm{H} \alpha$ and $\mathrm{Ca}$ II IRT (Liebert et al. 2003; Phan-Bao et al. 2006; Schmidt et al. 2007), coronal X-ray (Audard et al. 2007; Stelzer et al. 2006; Robrade \& Schmitt 2009), and radio (Berger 2002; Phan-Bao et al. 2007; Berger et al. 2008; Hallinan et al. 2008; Osten et al. 2009) emissions.

An alternative dynamo known as the so-called $\alpha^{2}$ dynamo maintained by convection alone was proposed (Roberts \& Stix 1972). It has been developed for fully convective pre-mainsequence stars (Schüssler 1975; Rüdiger \& Elstner 1994; Küker \& Rüdiger 1997, 1999) and late-M dwarfs and brown dwarfs (Dobler et al. 2006; Chabrier \& Küker 2006; Browning 2008). In general, these models predict large-scale fields, and magnetic field detections are now known for a number of active objects in these groups (Saar 1994; Johns-Krull \& Valenti 1996; Donati et al. 2006a; Morin et al. 2008; Berger et al. 2009). However, the models disagree with each other on the magnetic field morphology and they disagree with the results from some phaseresolved spectropolarimetric observations (Donati et al. 2006a; 
Table 1

Physical Parameters of the Six M Dwarfs

\begin{tabular}{|c|c|c|c|c|c|c|c|c|c|}
\hline Star & $\mathrm{SpT}^{\mathrm{a}}$ & $M_{\mathrm{K}}^{\mathrm{b}}$ & $\begin{array}{l}\log L_{\mathrm{bol}}{ }^{\mathrm{c}} \\
\left(\mathrm{erg} \mathrm{s}^{-1}\right)\end{array}$ & $\begin{array}{c}v \sin i^{\mathrm{d}} \\
\left(\mathrm{km} \mathrm{s}^{-1}\right)\end{array}$ & $\log \left(R_{\mathrm{X}}\right)^{\mathrm{e}}$ & $\begin{array}{c}M_{\star}^{\mathrm{f}} \\
\left(M_{\odot}\right)\end{array}$ & $\begin{array}{c}B_{\mathrm{Z}} \\
(\mathrm{G})\end{array}$ & $\begin{array}{c}\mathrm{H} \alpha \mathrm{EW} \\
(\AA)\end{array}$ & $\begin{array}{c}B_{\mathrm{Z}}(\mathrm{H} \alpha) \\
(\mathrm{G})\end{array}$ \\
\hline Gl 890 & M0 & 5.41 & 32.26 & 70 & -3.26 & 0.57 & $<18$ & $0.64 \pm 0.01$ & $<22$ \\
\hline LHS 473 & M2.5 & 5.83 & 32.09 & $<2.6$ & -4.98 & 0.48 & $16 \pm 7$ & $-0.29 \pm 0.02$ & $<30$ \\
\hline KP Tau & M3 & 6.82 & 31.66 & 32 & -3.53 & 0.31 & $\begin{array}{r}-104 \pm 32 \\
-92 \pm 32\end{array}$ & $\begin{array}{l}0.73 \pm 0.05 \\
0.73 \pm 0.05\end{array}$ & $\begin{array}{r}-580 \pm 220 \\
210 \pm 110\end{array}$ \\
\hline G 164-31 & M4 & 6.73 & 31.66 & 41 & -2.61 & 0.33 & $680^{\mathrm{g}}$ & $3.15 \pm 0.01^{\mathrm{g}}$ & $530 \pm 60^{g}$ \\
\hline G1 896B & M4.5 & 7.28 & 31.45 & 24.2 & -3.26 & 0.25 & $\begin{array}{l}296 \pm 40 \\
294 \pm 37 \\
279 \pm 38\end{array}$ & $\begin{array}{l}3.5 \pm 0.3 \\
3.6 \pm 0.3 \\
4.8 \pm 0.5\end{array}$ & $\begin{array}{r}-150 \pm 60 \\
110 \pm 60 \\
160 \pm 15\end{array}$ \\
\hline $2 \mathrm{E} 4498$ & M4.5 & 7.60 & 31.31 & 55 & -2.46 & 0.21 & $-440 \pm 84$ & $4.7 \pm 0.5$ & $-270 \pm 100$ \\
\hline
\end{tabular}

Notes.

a Spectral type from the literature.

b Absolute $K$-band magnitudes computed from trigonometric parallaxes (G1 890, LHS 473, G 164-31, and G1 896B) or spectroscopic distances (KP Tau and 2E 4498) and 2MASS $K$-band magnitudes available in the Vizier database.

${ }^{\mathrm{c}}$ Bolometric luminosity estimated using the bolometric correction $B C_{\mathrm{K}}$ vs. $K$-band magnitude relationship in Tinney et al. (1993).

${ }^{\mathrm{d}}$ Rotational velocity, see the text (Section 2.1) for references, except G 164-31 (this paper).

e $R_{\mathrm{X}}=L_{\mathrm{X}} / L_{\mathrm{bol}}$

${ }^{\text {f }}$ Stellar masses estimated using the $M_{\mathrm{K}}$ vs. mass empirical relationship in Delfosse et al. (2000).

${ }^{\mathrm{g}}$ For G 164-31: the value listed for an average magnetic flux $B f_{\mathrm{c}}$ computed using the ZDI technique (see Section 4.3 ), assuming $f_{\mathrm{c}}=1$. Its $\mathrm{H} \alpha$ equivalent width and chromospheric field strength $B_{\mathrm{Z}}$ are estimated at cycle $E=0.9181$ where the Zeeman signature appears very strong (see Figure 3).

Morin et al. 2008). For example, the latest dynamo simulations (Browning 2008) successfully predict that the magnetic fields possess large-scale fields with a lack of differential rotation in rapidly rotating $M$ dwarfs. However, the simulations predict the field morphology in fully convective stars should be mostly toroidal, whereas results of Donati et al. (2006a) and Morin et al. (2008) show dominantly poloidal fields in five M3-M4.5 dwarfs.

In this paper, we present our mapping of the M4 dwarf G 16431 based on $20 \mathrm{hr}$ of spectropolarimetric observations spread over three successive nights. To date, there are only two M4 dwarfs (V374 Peg, Donati et al. 2006a; G 164-31, this paper) that have been densely monitored over only a few rotation cycles so as to preclude intrinsically long-term magnetic variability, which can affect the mapping result. In addition, for the first time a synthetic spectrum fitting technique (Marcy 1982; Saar 1988; Johns-Krull \& Valenti 1996) is applied for measuring the mean field strength and the filling factor in G 164-31. Together, the polarimetric field mapping and the mean field measurements provide us a better accounting of both large- and small-scale fields in one of these two rapid rotators. We also present our Zeeman signature search in Stokes $V$ for five other $\mathrm{M}$ dwarfs with spectral types ranging from M0 to M4.5: Gl 890 (M0V), LHS 473 (M2.5V), KP Tau (M3V), Gl 896B (M4.5V), and 2E 4498 (M4.5V). Strong circular polarization is detected in KP Tau, G1 896B, and 2E 4498.

We present our targets in Section 2 and the observation and the data reduction in Section 3. We apply the tomographic imaging technique for constructing the field topology of Gl 490B in Section 4. We discuss the field topology of the six M dwarfs in Section 5 and summarize the results in Section 6.

\section{TARGETS}

\subsection{Targets}

All the six targets have spectral types from M0V to M4.5V and their physical parameters are listed in Table 1. From our mass estimates (see Table 1 and references therein), three $M$ dwarfs
(KP Tau, G 164-31, and 2E 4498) have masses estimated below $0.35 M_{\odot}$ (or absolute magnitudes fainter than $M_{\mathrm{K}}=6.62$ ) and are expected to be fully convective according to standard models. Two early-M dwarfs (Gl 890 and LHS 473) are partially convective. The absolute magnitude of the unresolved binary system Gl $896 \mathrm{Bab}$ is $M_{\mathrm{K}}=7.28$, which gives $M_{\mathrm{K}}>7.28$ for each companion. Both components are therefore expected to be fully convective.

\subsubsection{Gl 890 (HK Aqr), MOV}

This flaring M0 dwarf is a rapid rotator with $v \sin i=$ $70 \mathrm{~km} \mathrm{~s}^{-1}$ (Young et al. 1984). A photometric period of 0.431 days was determined by Young et al. (1990). The star has been well studied for magnetic activity, but no circular polarization observations have been reported so far. Rao \& Singh (1990) detected a steady soft X-ray emission from the source, indicating coronal activity. Its X-ray luminosity $(0.2-$ $4.5 \mathrm{keV})$ was measured by XMM-Newton at $\log L_{\mathrm{X}}\left(\mathrm{erg} \mathrm{s}^{-1}\right)$ $\approx 29.0$ (XMM-SSC 2008). A large flare-like event was also detected by Singh et al. (1999) from the X-ray light curve. Gl 890 was observed with the Australia Telescope Compact Array (ATCA) at $6 \mathrm{~cm}$ over the full rotation phase in 1990, but the source was not detected at an upper flux limit of $\approx 0.3 \mathrm{mJy}$ (Lim 1993). It was subsequently detected as a weak (below $0.3 \mathrm{mJy}$ ) slowly varying source with the Very Large Arra (VLA; Lim 1993, quoting a private communication by M. Güdel \& A. D. Benz 1992).

\subsubsection{LHS $473($ GJ 752A), M2.5V}

LHS 473 is an M2.5 dwarf, a companion of VB 10 (M8), and has a low rotational velocity $v \sin i<2.6 \mathrm{~km} \mathrm{~s}^{-1}$ (Delfosse et al. 1998). Vaiana et al. (1981) reported a soft X-ray (0.2$4.0 \mathrm{keV}$ ) detection with $\log L_{\mathrm{X}}=27.11$. Leto et al. (2000) carried out VLA radio observations but the star was not detected in all four different observed radio bands. These observations indicate a low coronal activity level in LHS 473. Stauffer \& Hartmann (1986) also reported weak chromospheric activity with an $\mathrm{H} \alpha$ absorption equivalent width of $0.35 \AA$ (Cram \& 
Giampapa 1987). Johns-Krull \& Valenti (1996) classified LHS 473 an inactive $\mathrm{M}$ dwarf.

\subsubsection{KP Tau (Wolf 1246, RX J0339.4+2457), M3V}

This M3 dwarf, in contrast with LHS 473, is a rapid rotator with $v \sin i=32 \mathrm{~km} \mathrm{~s}^{-1}$ (Mochnacki et al. 2002). The dwarf is a ROSAT source $(0.1-2.4 \mathrm{keV})$ with $\log L_{\mathrm{X}}=28.13$ (Fleming 1998; Hünsch et al. 1999), implying that the coronal activity level in KP Tau is over an order of magnitude higher than that in LHS 473 (which has a similar spectral type). To date, no radio observations of the star have been reported.

\subsubsection{G 164-31 (Gl 490B), $M 4 \mathrm{~V}$}

G 164-31 is a star with a spectral type of M4V (Reid et al. 1995) at a distance of $18.1 \mathrm{pc}$ (ESA 1997), which is the companion of $\mathrm{G} \mathrm{164-32} \mathrm{(Gl} \mathrm{490A,} \mathrm{M0.5V)} \mathrm{at} \mathrm{a} \mathrm{large}$ angular separation of $15^{\prime \prime}$. At the given distance and its apparent Two Micron All Sky Survey (2MASS) $K$-band magnitude of $K=8.02$, we derive its absolute magnitude $M_{\mathrm{K}}=6.73$. This fast rotator with $v \sin i=34 \mathrm{~km} \mathrm{~s}^{-1}$ exhibits high coronal activity in X-ray emission (0.3-3.5 keV) with $\log L_{\mathrm{X}}=29.05$ (Fleming et al. 1989). No radio observations of the star have been reported to date. Using the Segransan et al. (2003) empirical relationship of $M_{\mathrm{K}}$ versus radius, we derive $R_{\star}=0.34 R_{\odot}$, which is in good agreement with theoretical models (e.g., Baraffe et al. 1998) for inactive dwarfs (see Demory et al. 2009). This estimated radius yields a maximum rotational period of $\sim 12.1 \mathrm{hr}$.

\subsubsection{Gl 896B (EQ Peg B), M4.5V}

The Gl 896 system is a quadruple Gl 896AabBab (Delfosse et al. 1999; Oppenheimer et al. 2001). Gl 896 Bab is a singlelined spectroscopic binary with a joint spectral type of M4.5 and a joint $v \sin i$ of $24.2 \mathrm{~km} \mathrm{~s}^{-1}$ (Delfosse et al. 1998). Gl 896Bab was detected at radio frequencies (Güdel et al. 1993) of $4.9 \mathrm{GHz}$ $(6 \mathrm{~cm})$ and $8.5 \mathrm{GHz}(3.6 \mathrm{~cm})$. The joint X-ray luminosity $(0.1-$ $2.4 \mathrm{keV}$ ) of the $\mathrm{Gl} 896 \mathrm{AabBab}$ system is $\log L_{\mathrm{X}}=28.84$ (Güdel et al. 1993). Robrade \& Schmitt (2009) reported Gl 896Bab was on average a factor of 3.5 weaker than Gl 896Aab, we therefore derive $\log L_{\mathrm{X}}=28.19$ for Gl 896Bab.

\subsection{6. $2 E 4498$ (RX J2137.6+0137), M4.5V}

No spectral type estimate of this dwarf is available in the literature. From our unpolarized (Stokes $I$ ) spectra obtained with ESPaDOnS (see Section 3.1), using a spectral index versus spectral type relationships for M dwarfs (e.g., Martín et al. 1999), we estimate its spectral type as M4.5 \pm 0.5 , giving it an absolute magnitude $M_{\mathrm{I}} \approx 10.1$ (e.g., Leggett 1992). Comparison with the DENIS apparent magnitude $I=10.38$ (Epchtein 1997) then gives a distance of $\sim 11.4 \mathrm{pc}$, assuming no reddening. The source shows coronal activity in X-ray emission $(0.1-2.4 \mathrm{keV})$ with a measured flux $f_{\mathrm{X}}=4.52 \times 10^{-12} \mathrm{erg}$ $\mathrm{s}^{-1} \mathrm{~cm}^{-2}$ and $20 \mathrm{~cm}$ radio emissions (see Brinkmann et al. 2000 and references therein). At the source distance, we derive $\log L_{\mathrm{X}}=28.85$. The radius of an M4.5 dwarf is about $0.3 R_{\odot}$ (Chabrier \& Baraffe 1997), resulting in a maximum rotational period of $7.5 \mathrm{hr}$ at $v \sin i=55 \mathrm{~km} \mathrm{~s}^{-1}$ (Mochnacki et al. 2002). We detected strong circular polarization in this dwarf (see Figure 1). Since its rotation period is quite short, our detection makes the source $\left(M_{\star} \sim 0.2 M_{\odot}\right)$ an excellent target for future mapping of the magnetic field on a fully convective star.

\section{SPECTROPOLARIMETRIC OBSERVATIONS AND RESULTS}

\subsection{Observations}

We observed the six $M$ dwarfs described above with the Canada-France-Hawaii Telescope's ESPaDOnS highresolution spectrograph $(R=65,000$; Donati 2003), which provides a wavelength coverage of $370-1000 \mathrm{~nm}$. The spectropolarimetric mode was used to provide unpolarized (Stokes $I$ ) and circularly polarized (Stokes $V$ ) spectra. Among the six targets, $\mathrm{G}$ 164-31 is a particularly interesting source since it is an analog of V374 Peg (G 188-38), which is a rapidly rotating M4 dwarf with $v \sin i \approx 37 \mathrm{~km} \mathrm{~s}^{-1}$ showing a large-scale poloidal field (Donati et al. 2006a). G 164-31 is therefore also an excellent target on which to study the field topology in fully convective stars.

Each observation consists of four exposures taken at different polarimeter configurations and combined together to filter out spurious polarization signatures to first order (Donati et al. 1997). Exposure times were computed using the exposure time calculator for ESPaDOnS to obtain signal-to-noise ratios (S/Ns) above 100 at the wavelengths of interest. This signalto-noise value is typical of what is needed to detect the circular polarization in M dwarfs (e.g., Phan-Bao et al. 2006). In the case of G 164-31, we monitored the star for a total of $20 \mathrm{hr}$ from 2008 March 23 to 26 with 33 observations, aiming to cover at least one full rotation period of the star. The full observing logs are given in Table 2.

Data reduction was performed using Libre-ESpRIT (Donati et al. 1997) utilizing the principles of optimal extraction as given in Horne (1986). As the Zeeman signature is typically very weak, several methods (Semel et al. 2009; Martínez González et al. 2008; Donati et al. 1997) have been used in the past to increase the $\mathrm{S} / \mathrm{N}$ of Zeeman signatures. In this work, the least-squares deconvolution (LSD; Donati et al. 1997) multiline analysis procedure was applied to the spectra to extract the polarization signal from a large number of photospheric atomic lines ${ }^{8}$ and compute a mean Zeeman signature corresponding to an average photospheric profile centered at $700 \mathrm{~nm}$. Both mean Stokes $I$ and $V$ profiles are computed for all collected spectra. Figure 1 shows the LSD Stokes $I$ and $V$ profiles of KP Tau, Gl 896B, and 2E 4498 in which we detect Zeeman signatures. The profiles of G 164-31 are shown in Figures 2 and 3. Using the basic equations listed in (Wade et al. 2000; see also Brown \& Landstreet 1981; Donati et al. 1997), we compute the net longitudinal magnetic field strength, $B_{z}$, from our LSD Stokes $I$ and $V$ profiles. In the case of G 164-31, its time series of Stokes $I$ and Stokes $V$ profiles are used in the following section to reconstruct the field topology on this M4 dwarf.

\subsection{Results}

Table 1 lists our measurements of $B_{\mathrm{Z}}$ for all targets. We detected strong Zeeman signatures in KP Tau, G164-31, Gl 896B, and 2E 4498 but we did not detect them in the two early-M dwarfs Gl 890 and LHS 473. The photospheric field

\footnotetext{
8 We used a line list (Kurucz 1993) corresponding to M spectral types matching that of the six dwarfs. Approximately 5000 intermediate to strong atomic spectral lines with an average Landé factor $g_{\text {eff }}$ of $\sim 1.2$ are used simultaneously to retrieve the average polarization information with typical noise levels of $\approx 0.08 \%$ (relative to the unpolarized continuum level) per $1.8 \mathrm{~km} \mathrm{~s}^{-1}$ velocity bin and per individual polarization spectrum. This represents a multiplex gain in $\mathrm{S} / \mathrm{N}$ of about 10 with respect to a single line analysis.
} 
Table 2

Observing Logs for the Six M Dwarfs

\begin{tabular}{|c|c|c|c|c|c|c|}
\hline Target & Date & $\begin{array}{c}\text { HJD }^{\mathrm{a}} \\
(2453000+) \\
\end{array}$ & $\begin{array}{c}\text { UT } \\
\text { (h:m:s) }\end{array}$ & $\begin{array}{l}\text { Exposure } \\
\text { time (s) }\end{array}$ & $\begin{array}{c}\text { Peak of } \\
\text { S/N }\end{array}$ & Cycle $^{b}$ \\
\hline G1 890 & 2007 Sep 29 & 1372.91386 & $09: 49: 13$ & $4 \times 200$ & 166 & \\
\hline LHS 473 & 2005 Sep 18 & 631.74709 & $05: 52: 12$ & $4 \times 300$ & 511 & \\
\hline \multirow[t]{2}{*}{ KP Tau } & 2007 Sep 29 & 1373.05187 & 13:08:24 & $4 \times 500$ & 153 & $\ldots$ \\
\hline & 29 & 1373.07920 & $13: 47: 45$ & $4 \times 500$ & 153 & \\
\hline \multirow[t]{3}{*}{ Gl 896B } & 2007 Sep 29 & 1372.93533 & $10: 18: 07$ & $4 \times 300$ & 182 & $\ldots$ \\
\hline & 29 & 1372.95603 & $10: 47: 55$ & $4 \times 400$ & 209 & $\ldots$ \\
\hline & 29 & 1373.02336 & $12: 24: 53$ & $4 \times 400$ & 195 & \\
\hline $2 \mathrm{E} 4498$ & 2007 Sep 29 & 1372.89021 & $09: 15: 32$ & $4 \times 500$ & 139 & \\
\hline \multirow[t]{33}{*}{ G 164-31 } & 2008 Mar 24 & 1549.80662 & $07: 16: 47$ & $4 \times 500$ & 140 & 0.4937 \\
\hline & 24 & 1549.83227 & $07: 53: 43$ & $4 \times 500$ & 144 & 0.5412 \\
\hline & 24 & 1549.85770 & 08:30:19 & $4 \times 500$ & 145 & 0.5883 \\
\hline & 24 & 1549.88332 & 09:07:13 & $4 \times 500$ & 143 & 0.6358 \\
\hline & 24 & 1549.90865 & 09:43:42 & $4 \times 500$ & 140 & 0.6827 \\
\hline & 24 & 1549.93419 & $10: 20: 28$ & $4 \times 500$ & 141 & 0.7300 \\
\hline & 24 & 1549.95967 & $10: 57: 10$ & $4 \times 500$ & 144 & 0.7772 \\
\hline & 24 & 1549.98510 & $11: 33: 47$ & $4 \times 500$ & 145 & 0.8243 \\
\hline & 24 & 1550.01040 & $12: 10: 13$ & $4 \times 500$ & 145 & 0.8711 \\
\hline & 24 & 1550.03576 & $12: 46: 44$ & $4 \times 500$ & 144 & 0.9181 \\
\hline & 24 & 1550.06109 & $13: 23: 13$ & $4 \times 500$ & 149 & 0.9650 \\
\hline & 24 & 1550.08637 & $13: 59: 36$ & $4 \times 500$ & 150 & 1.0118 \\
\hline & 25 & 1550.87304 & $08: 52: 24$ & $4 \times 500$ & 132 & 2.4686 \\
\hline & 25 & 1550.89882 & 09:29:31 & $4 \times 500$ & 138 & 2.5163 \\
\hline & 25 & 1550.92424 & 10:06:08 & $4 \times 500$ & 144 & 2.5634 \\
\hline & 25 & 1550.94977 & $10: 42: 53$ & $4 \times 500$ & 137 & 2.6107 \\
\hline & 25 & 1550.97519 & 11:19:29 & $4 \times 500$ & 140 & 2.6578 \\
\hline & 25 & 1551.00055 & 11:56:01 & $4 \times 500$ & 149 & 2.7047 \\
\hline & 25 & 1551.02679 & $12: 33: 48$ & $4 \times 500$ & 144 & 2.7533 \\
\hline & 25 & 1551.05210 & $13: 10: 15$ & $4 \times 500$ & 145 & 2.8002 \\
\hline & 25 & 1551.07741 & $13: 46: 41$ & $4 \times 500$ & 135 & 2.8471 \\
\hline & 25 & 1551.10286 & $14: 23: 20$ & $4 \times 500$ & 145 & 2.8942 \\
\hline & 26 & 1551.92059 & 10:00:51 & $4 \times 464$ & 138 & 4.4085 \\
\hline & 26 & 1551.94449 & $10: 35: 15$ & $4 \times 464$ & 139 & 4.4528 \\
\hline & 26 & 1551.96815 & 11:09:20 & $4 \times 464$ & 144 & 4.4966 \\
\hline & 26 & 1551.99187 & $11: 43: 30$ & $4 \times 464$ & 127 & 4.5405 \\
\hline & 26 & 1552.01563 & $12: 17: 42$ & $4 \times 464$ & 132 & 4.5845 \\
\hline & 26 & 1552.04024 & $12: 53: 09$ & $4 \times 464$ & 134 & 4.6301 \\
\hline & 26 & 1552.06392 & $13: 27: 14$ & $4 \times 464$ & 141 & 4.6739 \\
\hline & 26 & 1552.08759 & $14: 01: 20$ & $4 \times 464$ & 134 & 4.7178 \\
\hline & 26 & 1552.11210 & $14: 36: 37$ & $4 \times 464$ & 132 & 4.7631 \\
\hline & 26 & 1552.13587 & $15: 10: 51$ & $4 \times 464$ & 114 & 4.8072 \\
\hline & 27 & 1553.08983 & $14: 04: 32$ & $4 \times 464$ & 135 & 6.5738 \\
\hline
\end{tabular}

Notes.

${ }^{\text {a }}$ Heliocentric Julian date (UTC).

b Rotational cycles $E$ are computed using ephemeris HJD $=2454549.54+$ $0.54 E$, only available for $\mathrm{G} 164-31$

strength is estimated from LSD Stokes $I$ and $V$ profiles, and the chromospheric field is computed from the Stokes profiles of only the $\mathrm{H} \alpha$ line.

For G 164-31, we find that the Zeeman signatures repeat after $0.540 \pm 0.006$ days $(12.96 \pm 0.14 \mathrm{hr})$, which is in the expected range of its rotation period. We thus conclude that the rotation period of this dwarf is $12.96 \mathrm{hr}$. We note that this value is slightly greater than the estimated maximum rotation period of $12.1 \mathrm{hr}$ for this star. The difference is possibly due to $\mathrm{G} 164-31$ having a larger radius than the expected value from theory. Matching unpolarized (Stokes $I$ ) spectra of G 164-31 with that of slowly rotating references requires a projected rotation velocity of $v \sin$ $i=41 \pm 1 \mathrm{~km} \mathrm{~s}^{-1}$. Our value is greater than one of Fleming et al. (1989), $v \sin i=34 \pm 8.5 \mathrm{~km} \mathrm{~s}^{-1}$, though both measurements are consistent to within the uncertainties. Dues to its smaller


Figure 1. LSD Stokes $V$ and $I$ profiles of three M dwarfs: KP Tau (a), Gl 896B (b), and 2E 4498 (c). Strong circular polarization is seen in the three stars.

uncertainty, we adopt our $v \sin i=41 \mathrm{~km} \mathrm{~s}^{-1}$ for $\mathrm{G} 164-31$. From $P_{\text {rot }}$ and $v \sin i$, we derive $R \sin i=0.44 \pm 0.02 R_{\odot}$. Our $R$ sin $i$ is $\sim 30 \%$ larger than the estimated stellar radius (see Section 2.1.4). It is unlikely that $i$ exactly equals $90^{\circ}$, and the results obtained in the imaging process described below are similar for $60^{\circ} \leqslant i \leqslant 80^{\circ}$. We thus set $i=70^{\circ}$, which results in the $\mathrm{G} 164-31$ radius being $\sim 38 \%$ larger than the typical value for $\mathrm{M}$ dwarfs of the same luminosity. This is likely due to the fact that $\mathrm{G} 164-31$ is a young object belonging to a moving cluster identified by Orlov et al. (1995). We refer the reader to Section 5 for further discussion.

We monitored G 164-31 for $20 \mathrm{hr}$ spread over three successive nights, aiming for coverage over a full rotational cycle. The true period $(12.96 \mathrm{hr}$ ) is a bit longer than our expected maximum value $(12.1 \mathrm{hr})$. With this period, our observations cover only half of the rotational phase. In other words, only half of the stellar surface was monitored. A few of the Stokes $I$ and $V$ LSD profiles are discarded due to the contamination of moonlight or flaring events. Figure 2 and 3 show Stokes $I$ and $V$ LSD profiles of G 164-31.

\section{MODELING OF MAGNETIC FIELD TOPOLOGY OF G 164-31}

To reconstruct the map of cool spots and magnetic fields at the surface of G 164-31 from the set of observed LSD Stokes I and $V$, we use the Donati et al. (2001) magnetic mapping code which employs the Zeeman-Doppler imaging (ZDI) technique (Semel et al. 1989). The typical longitude resolution achieved at 


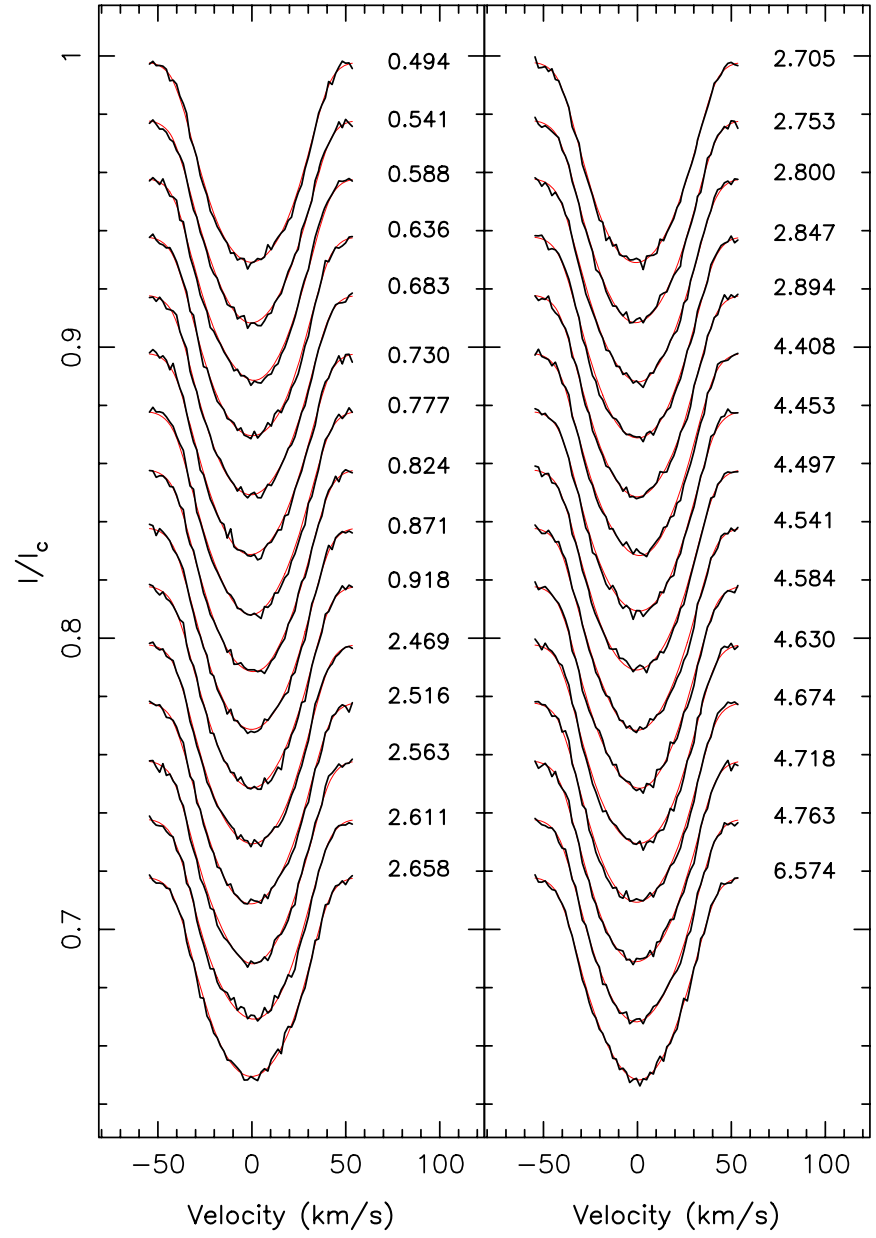

Figure 2. LSD Stokes $I$ profiles of G 164-31 (black line) along with the maximum entropy fits (red line) to the data. The rotational phases are indicated to the right of each profile.

(A color version of this figure is available in the online journal.)

the equator is $\approx 15^{\circ}$ or $\approx 0.04$ rotation cycle. The basic idea of the code has been fully described in several papers (Brown et al. 1991; Donati \& Brown 1997; Donati et al. 2001, 2006b; Morin et al. 2008). In this paper, we briefly describe the principles of the modeling work and refer the reader to the above papers for further details.

\subsection{Mapping Spots}

Cool spots on the surface of a rapidly rotating star produce distortions in the stellar spectral lines. If the star is rotating fast enough (at least 20-30 $\mathrm{km} \mathrm{s}^{-1}$ for late-type dwarfs; Vogt et al. 1987), the shape of the star's spectral line profiles is dominated by rotational Doppler broadening. In this case, there is a strong correlation between the position of any distortion within a line profile and the position of the corresponding spot on the stellar surface. A spectrum of the rapid rotator is a one-dimensional image that is resolved in the direction perpendicular to the stellar rotation axis and the light of sight. By observing the star at different rotation phases, a two-dimensional image of the spot distribution can be reconstructed using the so-called Doppler imaging (DI) technique (see Vogt et al. 1987 and references therein). In the imaging process, the stellar surface is divided into a grid of 1000 elementary cells. Using the spot-occupancy model of Cameron (1992), the local line profile at each grid point of the surface is described as a linear combination of two

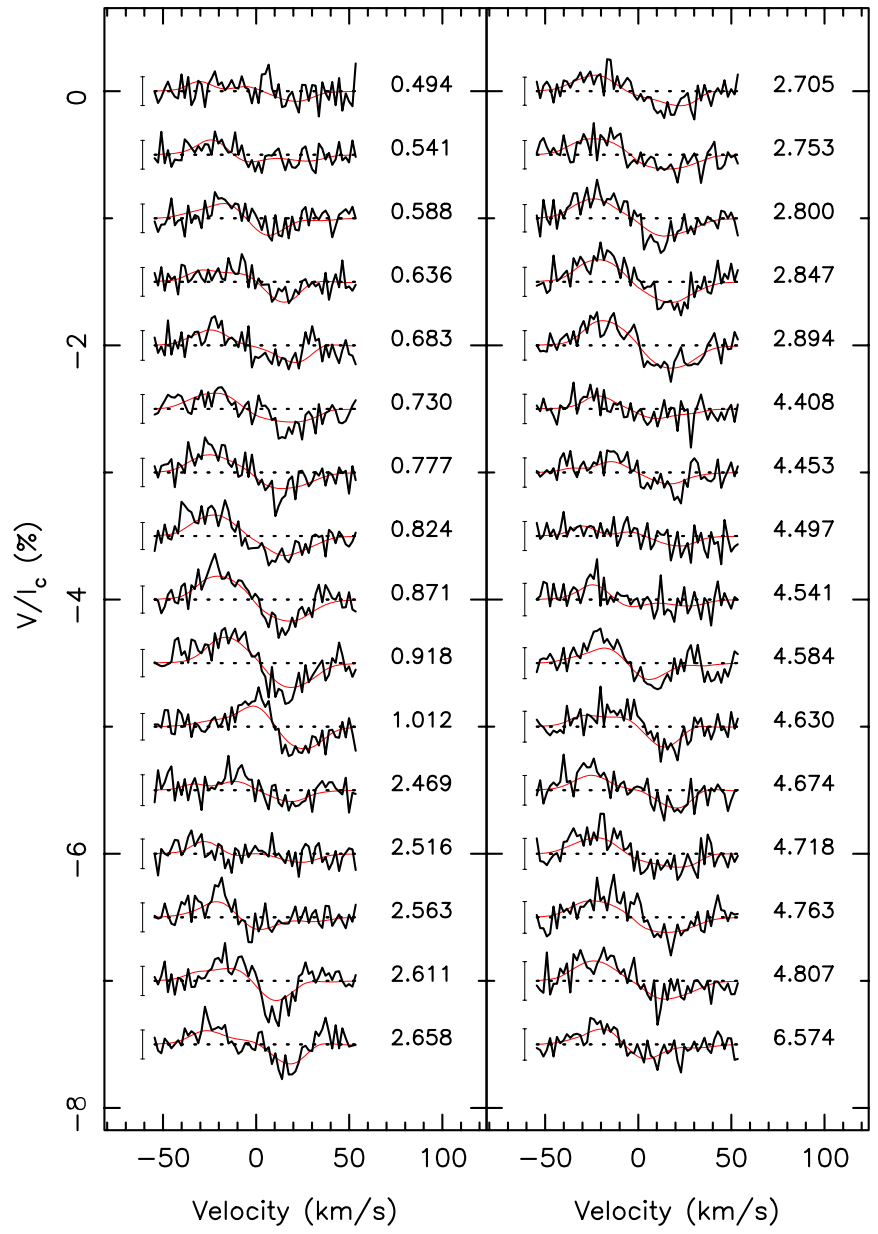

Figure 3. LSD Stokes $V$ profiles of G 164-31 (black line) along with the maximum entropy fits (red line) to the data. The rotational phase (right) and the $3 \sigma$ error bar (left) of each profile are shown accordingly.

(A color version of this figure is available in the online journal.)

reference profiles, one representing the quiet photosphere and the other for cool spots. Both reference profiles are assumed to be equal and only differ by their relative continuum levels. For the assumed reference profile, we can use either the LSD profile of the very slowly rotating inactive M4 dwarf G1 402 or a simple Gaussian profile with similar full width at half-maximum and equivalent width. Both options yield very similar results, indicating that the exact shape of the assumed local profiles has very minor impact on the reconstructed images, as long as the rotational velocity of the star is much larger than the local profile. Each element on the surface is quantified by the local fraction occupied by spots. The spot occupancy ranges from 0 (no spots) to 1 (complete spot coverage). The maximum entropy image reconstruction technique (Skilling \& Bryan 1984; Vogt 1980 ) is used to find the image having the smoothest variation (or in some sense the simplest image), giving the least contrast between spots and the quiet photosphere.

\subsection{Mapping Magnetic Fields}

The field is described as the sum of a poloidal and a toroidal component, both expressed as spherical harmonics expansions (Jardine et al. 1999). For a given set of the complex coefficients of the spherical harmonic expansions, one can produce a corresponding topology (or a Stokes $V$ data set) at the stellar surface. The code uses the maximum entropy image 
reconstruction technique, in which entropy (i.e., quantifying the amount of reconstructed information) is calculated from the coefficients of the spherical harmonics expansions. The imaging process starts from a null magnetic field and iteratively adjusts the spherical harmonic coefficients in order to match the synthetic Stokes $V$ profile to the observed LSD one. The code uses a multidirectional search in the image space until the required maximum entropy image is obtained. This corresponds to an optimal field topology that reproduces the data at a given $\chi^{2}$ level, i.e., usually down to noise level. Since the inversion problem is partially ill posed, we use the entropy function to select the magnetic field map with lowest information content among all those reproducing the data equally well.

To calculate the synthetic Stokes $V$ profiles for a given field topology, the surface was again divided into a grid of 1000 elementary surface cells. In each cell, the three components (radial, azimuthal, and meridional in spherical coordinates) of the vector field are estimated directly from the spherical harmonic expansion. The code uses the analytic solutions (Landolfi \& Landi Degl'Innocenti 1982) of Unno-Rachkovsky's radiative transfer equations to calculate the contribution to the Stokes $V$ profiles of all visible cells at each observed rotation phase. The free parameters in the Unno-Rachkovsky equations are obtained by fitting the LSD Stokes $I$ profile of a very slowly rotating and weakly active star with a similar spectral type (e.g., Gl 402). To obtain the best fit (in both amplitude and width) between the synthetic and observed Stokes $V$ profiles, the code introduces a filling factor $f_{\mathrm{c}}$, which represents the fractional amount of circular flux being constant over the whole stellar surface, and speculates that large-scale fields in $\mathrm{M}$ dwarfs can be structured on a small scale (Donati et al. 2008). The circularly polarized flux from each cell is thus $f_{\mathrm{c}} V_{\text {loc }}$, where $V_{\text {loc }}$ is the Stokes $V$ profile derived from the analytic solutions of the Unno-Rachkovsky equations.

Differential rotation is also implemented in the calculation process of the synthetic Stokes $V$ profiles for yielding the best fits to the observation. For this purpose, we assume the rotation rate varies with latitude, $\theta$, following a solar-like differential rotation law as $\Omega(\theta)=\Omega_{\mathrm{eq}}-d \Omega \sin ^{2} \theta$, where $\Omega_{\mathrm{eq}}$ is the angular rotation rate at the equator and $d \Omega$ is the difference in angular rotation rate between the equator and the pole. Differential rotation is detected when $\chi^{2}$ of the fit to the data shows a well-defined minimum in the range of $\Omega_{\mathrm{eq}}$ and $d \Omega$ values. In the case of $G$ 164-31, we did not obtain a clear minimum in the explored $\Omega_{\mathrm{eq}}-d \Omega$ range. This indicates that our data are not suitable for measuring differential rotation given the fairly simple (and low amplitude) rotational modulation of the Stokes $V$ profiles we observe. This is mostly due to the fact that the field distribution lacks well-defined features at different latitudes from which differential rotation can be estimated. Based on the previous observations of analogs of $\mathrm{G}$ 164-31 (Donati et al. 2006a; Morin et al. 2008), we therefore assumed that this star rotates as a rigid body while performing the field mapping. The obtained map of G 164-31 is shown in Figure 4.

\subsection{Results}

Figure 4 presents our maps of the spot occupancy and the magnetic field at the stellar surface of $G$ 164-31. For the half stellar surface that was not observed, the field topology has been derived using a spherical harmonic expansion based on the collected Stokes $V$ data set. Our brightness map shows a low contrast spot at the pole of the star. The magnetic field morphology on G 164-31 shows that the radial field component
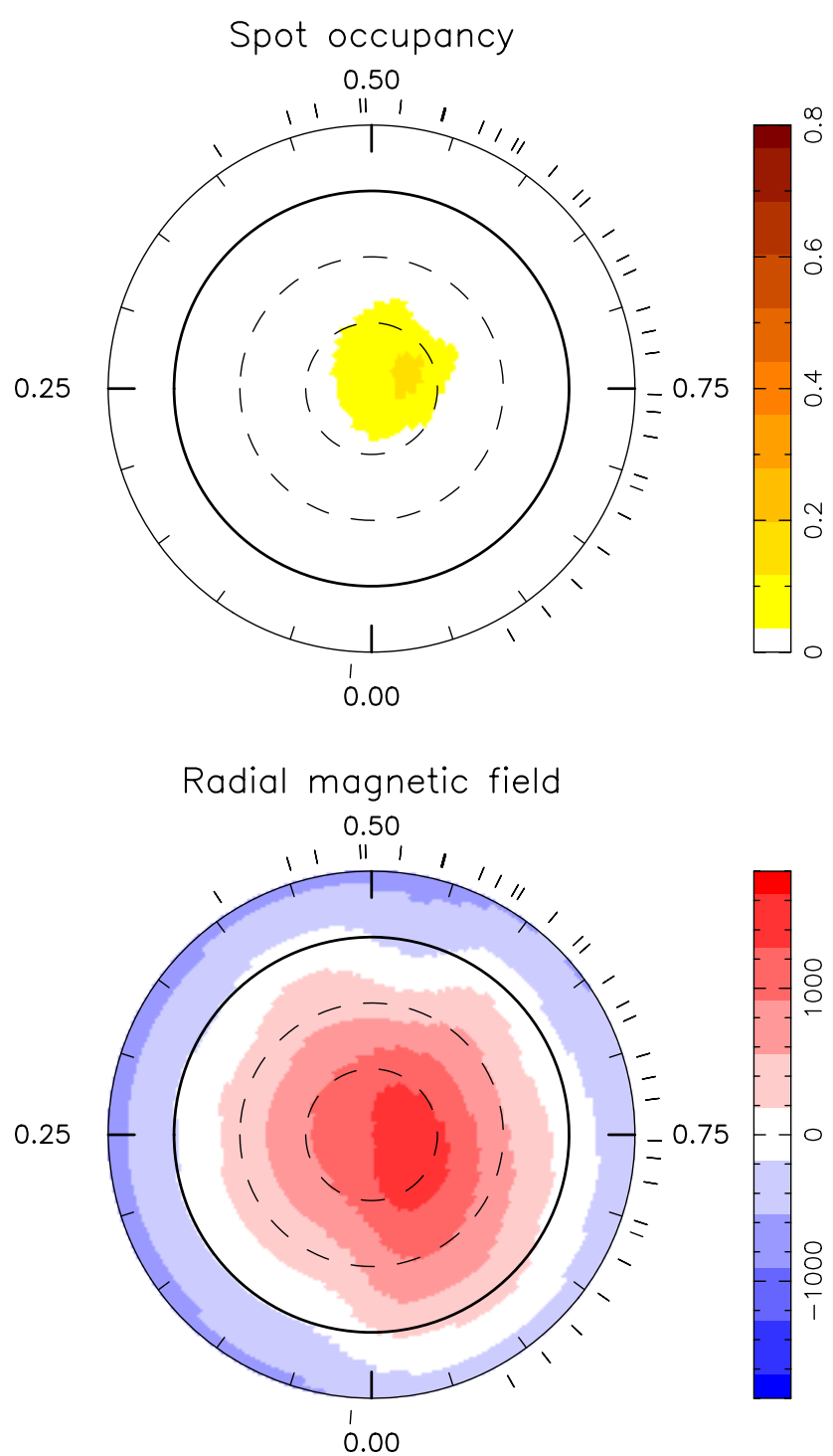

Figure 4. Magnetic field topologies of G 164-31, reconstructed from the series of LSD Stokes $V$ profiles. The radial field (bottom) dominates in the dwarf (intensity scale in Gauss), the azimuthal and meridional component are negligible. The spottedness (top) at the stellar surface is also reconstructed, the color scale represents spot occupancy for the dwarf $(1=$ complete spot coverage). The dwarf is shown in flattened polar projection extending down to latitudes of $-30^{\circ}$. The equator is described as a bold circle. Radial ticks outside the plots indicate the phases at which the dwarf was observed, a half of the dwarf surface had properly been monitored.

(A color version of this figure is available in the online journal.)

is dominant, which appears similar to what is seen in V374 Peg. The azimuthal and meridional components are negligible.

The average large-scale flux is $680 \mathrm{G}$ and the poloidal field dominates with $99 \%$ of the energy content. Most (95\%) of this poloidal field is stored in the mode corresponding to a dipole aligned with the rotation axis (spherical harmonics degree $l=1$ and order $m=0$ ). One should note that due to the high $v \sin$ $i$ of $\mathrm{G}$ 164-31, for any value of the filling factor $0<f_{\mathrm{c}}<1$, we find similar results. Hence, we arbitrarily set $f_{\mathrm{c}}=1$. We also examine the binarity of G 164-31. Based on the LSD Stokes $I$ profiles, we find an average radial velocity value of $-6.7 \pm 0.2 \mathrm{~km} \mathrm{~s}^{-1}$. No significant variation in radial velocity is found. Therefore, our current data do not reveal any hint of an additional close-in component around G 164-31. 


\section{DISCUSSION}

In this section, we first briefly discuss the magnetic field properties of the stars whose fields are not mapped. These stars are Gl 890, LHS 473, KP Tau, Gl 896B, and 2E 4498. We then focus the discussion on the field topology in the photosphere and the chromosphere of G 164-31.

\subsection{Individual Stars: Gl 890, LHS 473, KP Tau, Gl 896B, and $2 E 4498$}

We did not detect Zeeman signatures in Gl 890 and LHS 473. For the latter case, the non-detection in both the photosphere and the chromosphere indicates that LHS 473 is inactive, likely due to its low $v \sin i$, and this result is consistent with previous observations showing its low coronal activity level. However, in the fast rotating case of $\mathrm{Gl} 890$, the non-detection suggests that the field topology on this star may be different from what has been observed in more slowly rotating early-M dwarfs (Donati et al. 2008), whose fields are large-scale and dominantly toroidal producing Stokes $V$ signatures detectable at any time. Since the detections of X-ray and radio emission indicate that Gl 890 is magnetically active, phase-resolved spectropolarimetric observations of this star are therefore needed to clarify its magnetic field topology.

For the three remaining $\mathrm{M}$ dwarfs, all these fast rotators show Zeeman signatures, and they are particularly strong in Gl 896B and 2E 4498 (Figure 1). This result indicates the presence of strong, large-scale fields on these stars. Since KP Tau $\left(0.31 M_{\odot}\right)$ and $2 \mathrm{E} 4498\left(0.21 M_{\odot}\right)$ are fully convective, they are good targets for studying the field morphology by carrying out spectropolarimetric and simultaneous multiple-wavelength observations (e.g., Berger et al. 2009). We note that Gl 896Bab is an unresolved binary. Therefore, its Zeeman signature is contributed by two components if both are magnetically active.

\section{2. $G$ 164-31}

The G 164-31 magnetic field topology, which is mostly poloidal with most of the magnetic energy concentrated within the lowest order axisymmetric modes, is very similar to what has been observed in V374 Peg (Donati et al. 2006a) and other mid$\mathrm{M}$ dwarfs (Morin et al. 2008). The average large-scale magnetic flux on $\mathrm{G} 164-31$ is $0.68 \mathrm{kG}$, which is significantly smaller than the previous measurements of few kilogauss magnetic fluxes in active mid-M dwarfs using synthetic spectrum fitting techniques (e.g., Johns-Krull \& Valenti 1996). This is possibly due to the ZDI technique being sensitive to only large-scale and simple structures, while Zeeman signatures from magnetic regions with complicated or small-scale topology may cancel each other in circularly polarized spectra whereas these signatures add up in unpolarized spectra. To explore this "missing" magnetic flux, we used the synthetic spectrum fitting technique described in Johns-Krull \& Valenti (1996) to measure the mean magnetic flux on the surface of G 164-31. Briefly, spectra in the wavelength interval around the Zeeman-sensitive $\mathrm{Fe} I$ line at 8468.40 $\AA$ are analyzed. In stars as cool as G 164-31, this line is actually blended with a Ti I line at $8468.47 \AA$ which is similar in strength and also Zeeman sensitive. The spectrum synthesis models both lines plus numerous $\mathrm{TiO}$ lines in this wavelength region. Because of the ubiquitous $\mathrm{TiO}$, spectral changes due to magnetic fields are seen more clearly in the ratio of active to inactive line profiles. Therefore, spectra of G 164-31 were divided by inactive references. In our case, we used two inactive

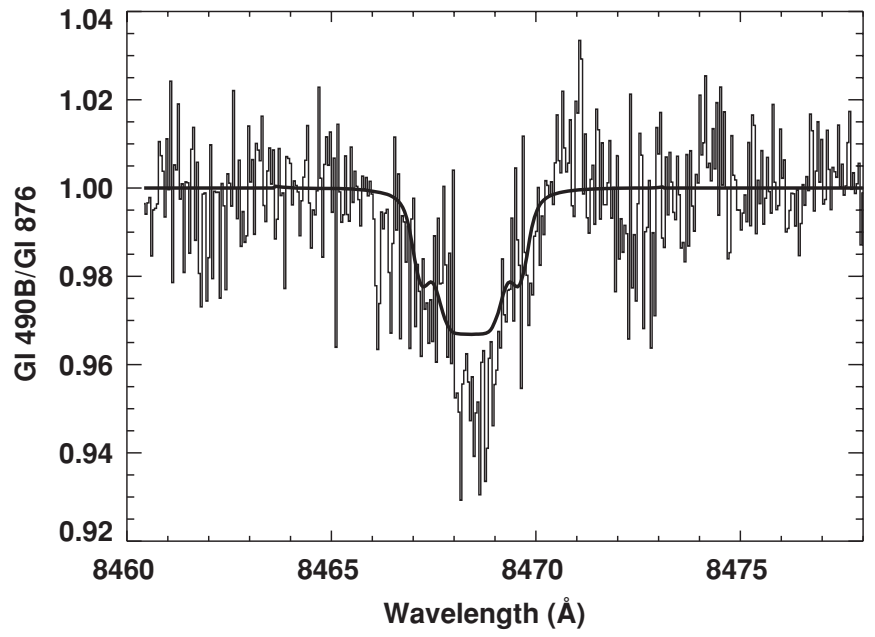

Figure 5. Best-fit modeling ratio of active to inactive profiles (thick line). The observed line profile ratio constructed by dividing the spectrum of $\mathrm{G}$ 164-31 (Gl 490B) in the wavelength region around the magnetically sensitive Fe I line at $8468.4 \AA$ by that of the inactive M4 dwarf G1 876 is shown (thin line).

reference stars GJ 725B (or LHS 59, M3.5V) and GJ 876 (or LHS 530, M4V) which are meant to be identical in all respects ( $T_{\text {eff }}, \log g,[\mathrm{M} / \mathrm{H}]$, etc.) to $\mathrm{G} 164-31$ except for the field. Line profiles were synthesized with and without magnetic fields (see Johns-Krull \& Valenti 1996 and references therein) and the ratio of active to inactive profiles was fitted by adjusting the magnetic field strength, $B$, and its filling factor, $f$, in the model spectrum of $\mathrm{G}$ 164-31. The line profiles are generated assuming a uniform radial field everywhere with a uniform filling factor.

The best fits were obtained with $B f=3.1 \mathrm{kG}$ and $B f=$ $3.3 \mathrm{kG}$ for GJ 725B and GJ 876. The fit to the observations using GJ 876 as the inactive reference star is shown in Figure 5. Due to the high $v \sin i$ of G 164-31, we cannot measure $B$ and $f$ separately. The model fit in the line core is not as good as we would like, and is only slightly improved using GJ 725B as the reference star. We note though that if the radius of G 16431 is indeed larger than expected due to youth (see below), its gravity is likely somewhat lower than that of either inactive comparison star. Our spectrum synthesis indicates that the $8468 \AA$ feature should actually weaken somewhat at lower gravity, which would result in the discrepancy in the core getting worse by $\sim 0.005$ in the ratio. A possible way to correct for this would be to fit a distribution of magnetic field strengths on the stellar surface as has been done for M dwarfs (Johns-Krull \& Valenti 2000) and on T Tauri stars (e.g., Johns-Krull et al. 1999). The current fit (Figure 5) attempts to use a single field strength to fit both the wings and the core, whereas a distribution of magnetic fields gives more flexibility in fitting the entire profile and can result in bigger changes in the line equivalent width than produced when using only a single field value. We therefore adopt $B f=3.2 \pm 0.4 \mathrm{kG}$ for $\mathrm{G} 164-31$ as the best estimate from our single field fitting described above, noting that this may be a slight underestimate. This value for $B f$ is larger than the one measured from the ZDI technique by a factor of 4.7. Our result is consistent with the previous measurements from the literature (e.g., EV Lac; Johns-Krull \& Valenti 1996; Morin et al. 2008), suggesting that in active mid-M dwarfs a significant part of the magnetic energy is stored in small-scale structures. As found by Reiners \& Basri (2009), it appears that a large majority of the magnetic energy is stored in the small-scale field of G 164-31. Apparently, a successful dynamo model should be 




Figure 6. H $\alpha$ equivalent width light curve. The curve appears likely sinusoidal, a sinusoidal curve (dashed line) is also shown for a comparison. The first vertical tick at 0.19 in the HJD offset (HJD-2454549.79717) position indicates the bottom of the light curve obtained in the first night. The remaining ticks are drawn with a distance of $2 \times P_{\text {rot }}$ between two consecutive ticks where the star is observable, where $P_{\text {rot }}=0.54$ days is the rotational period determined from the Stokes $V$ profile set (see Section 3.2). The observed bottoms of the light curve likely repeat after $2 P_{\text {rot }}$, suggesting that the $\mathrm{H} \alpha$ emission is modulated by the stellar rotation. Flaring events were observed in the first (strong) and second night (moderate). The decay and enhancement of $\mathrm{H} \alpha$ emission were also observed in the third night at around HJD offset $=2.1$ and 2.35, respectively This is possibly due to a decrease (or an increase) of the number of active regions. Changes in the $\mathrm{H} \alpha$ (also $\mathrm{H} \beta$ ) line profile shape (e.g., smoothness) seen in our spectra support this possibility.

able to produce both large-scale poloidal fields and small-scale features in rapidly rotating mid-M dwarfs.

To study chromospheric activity, we computed the $\mathrm{H} \alpha$ emission equivalent widths for each exposure. Our $\mathrm{H} \alpha$ emission light curve is shown in Figure 6. One should keep in mind that since each observation to obtain a Stokes $V$ profile consists of four exposures, measuring $\mathrm{H} \alpha$ emission at individual exposures therefore increases time resolution of the light curve. Figure 6 also indicates that the $\mathrm{H} \alpha$ equivalent width variations are likely sinusoidal and they are modulated by the stellar rotation with $P_{\text {rot }}=0.54$ days determined from the Stokes $V$ profiles. This effect has also been observed in late-M and brown dwarfs (see Berger et al. 2009 and references therein). Flaring events were seen strongly in the last sequences of the first observing night and moderately in the middle sequences of the second night. These events were also observed in the $\mathrm{H} \beta$ emission profiles. While the likely rotational modulation and nonzero minimum of the $\mathrm{H} \alpha$ light curve imply either a large-scale (poloidal or toroidal) chromospheric field or a localized concentration of small-scale magnetic activity, it is reasonable to assume that the chromospheric field in G 164-31 has both a large-scale poloidal component and small-scale structures as observed in the photospheric field. This field configuration probably dominates in both the photosphere and the chromosphere of the star. We note that simultaneous observations at multiple wavelengths of the source will place more constraints on the chromospheric and coronal field morphology (e.g., Berger et al. 2009).

It is very interesting to note that the radius of $G 164-31$ is at least $30 \%$ larger than that estimated from standard models for M dwarfs at the same luminosity (see Section 2.1.4). There are two possible interpretations of this discrepancy. First, the magnetic field effects of reduced convective efficiency due to fast rotation and large field strengths, and/or to spot coverage as proposed by Chabrier et al. (2007) might yield a cooler effective temperature $T_{\text {eff }}$ and thus a larger radius in low-mass stars, keeping their luminosity unchanged $\left(L \propto T_{\mathrm{eff}}^{4} R^{2}\right)$. Our spot mapping (Figure 4) shows the fraction of the stellar surface covered by the low contrast spot is relatively small in G 164-
31. However, one should note that the imaging technique is only sensitive to spots or spot groups with sizes comparable to our resolution element. We therefore cannot rule out the possibility of unresolved small spots spread everywhere on the stellar surface. Hence, we cannot conclude whether the effect due to spot coverage is significant in this star or not. Observational results (see Ribas 2006; Morales et al. 2008 and references therein) suggest that these effects might yield a $\sim 10 \%-15 \%$ larger radius in G 164-31, therefore the discrepancy in radius of over $30 \%$ cannot be explained by only those effects. Second, we explore the possibility that G 164-31 is a young M4 dwarf, its radius thus larger than one computed for old M dwarfs. We note that the $K$-band absolute magnitude of $\mathrm{G}$ $164-31, M_{\mathrm{K}}=6.73$, is 0.67 mag brighter than the average value for M4 dwarfs, ${ }^{9}$ supporting the young nature of the star. From a literature search, the binary system G 164-31 (Gl 490B)+G 164-32 (Gl 490A) indeed belongs to a moving cluster previously identified by (Orlov et al. 1995; for a review on young nearby stars, see Zuckerman \& Song 2004). One should note that the primary component $\mathrm{G}$ 164-32 also exhibits high coronal X-ray emission with $\log \left(L_{\mathrm{X}} / L_{\mathrm{bol}}\right)=-3.23$ (Fleming et al. 1989) and chromospheric $\mathrm{H} \alpha$ emission (Stauffer \& Hartmann 1986), indicating activity typical in young early-M dwarfs. We measured an upper limit for the $\mathrm{Li} \lambda 6708$ equivalent width of $17 \mathrm{~m} \AA$ for $\mathrm{G} 164-31$. This gives the star an age older than 10 Myr since early-M dwarfs $(\geqslant M 4)$ are expected to completely deplete their lithium within $\sim 10$ Myr. We suggest that G 164-31 is an analog of the M4 dwarf HIP $112312 \mathrm{~A}$ at $23.6 \mathrm{pc}$ (ESA 1997), which is a member of the $\sim 12$ Myr old $\beta$ Pictoris moving group (Song et al. 2002). To estimate the mass and age of G 16431, we used the Chabrier et al. (2007) and Baraffe et al. (1998) theoretical models with constraints of $M_{\mathrm{K}}=6.73, I-J=1.61$, and the deduced radius $R_{\star}=0.47 R_{\odot}$ of G 164-31, and we also considered the magnetic field effects that might yield about a $10 \%-15 \%$ larger radius. We then derived $M_{\star} \sim 0.15 M_{\odot}$ at an age of about 25-30 Myr, making G 164-31 the least massive $\mathrm{M}$ dwarf whose magnetic field has been mapped to date. More observations are needed to precisely determine the fundamental parameters of this moving cluster.

\section{SUMMARY}

Based on our circularly polarized and unpolarized spectroscopic observations, using both tomographic imaging and synthetic spectrum fitting, we reveal the mainly axisymmetric largescale poloidal magnetic field and the small-scale field structures storing a significant portion of the magnetic energy in the photosphere of the M4 dwarf G 164-31. The modulation of the $\mathrm{H} \alpha$ emission light curve suggests that the field in the chromosphere is stable and possibly poloidal like that in the photosphere. Our detection of circular polarization in the single and rapidly rotating M dwarfs KP Tau (M3V), 2E 4498 (M4.5V) makes them good targets for mapping of the field morphology in mid-M dwarfs in the future.

N.P.-B. has been aided in this work by a Henri Chretien International Research Grant administered by the American Astronomical Society. E.M. acknowledges support from the Spanish Ministry of Science through the project AYA200767458. The authors extend special thanks to those of Hawaiian

\footnotetext{
9 We selected nine single M4 dwarfs within $10 \mathrm{pc}$ listed in Delfosse et al. (1998) and computed their $K$-band absolute magnitude from trigonometric parallaxes and 2MASS $K$-band magnitudes available in the Vizier database. This work yielded an average value $M_{\mathrm{K}}=7.4$
} 
ancestry on whose sacred mountain we are privileged to be guests. Access to the CFHT was made possible by the Ministry of Education and the National Science Council of Taiwan as part of the Cosmology and Particle Astrophysics (CosPA) initiative.

\section{REFERENCES}

Audard, M., Osten, R. A., Brown, A., Briggs, K. R., Güdel, M., Hodges-Kluck, E., \& Gizis, J. E. 2007, A\&A, 471, L63

Baraffe, I., Chabrier, G., Allard, F., \& Hauschildt, P. H. 1998, A\&A, 337, 403

Berger, E. 2002, ApJ, 572, 503

Berger, E., et al. 2008, ApJ, 676, 1307

Berger, E., et al. 2009, ApJ, 695, 310

Brinkmann, W., Laurent-Muehleisen, S. A., Voges, W., Siebert, J., Becker, R. H., Brotherton, M. S., White, R. L., \& Gregg, M. D. 2000, A\&A, 356, 445

Brown, S., Donati, J.-F., Rees, D., \& Semel, M. 1991, A\&A, 250, 463

Brown, D. N., \& Landstreet, J. D. 1981, ApJ, 246, 899

Browning, M. K. 2008, ApJ, 676, 1262

Cameron, A. 1992, in Lecture Notes in Physics 397, Surface Inhomogeneities on Late-Type Stars, ed. P. B. Byrne \& D. J. Mullan (Berlin: Springer), 33

Chabrier, G., \& Baraffe, I. 1997, A\&A, 327, 1039

Chabrier, G., Gallardo, J., \& Baraffe, I. 2007, A\&A, 472, L17

Chabrier, G., \& Küker, M. 2006, A\&A, 446, 1027

Cram, L. E., \& Giampapa, M. S. 1987, ApJ, 323, 316

Delfosse, X., Forveille, T., Beuzit, J.-L., Udry, S., Mayor, M., \& Perrier, C. 1999, A\&A, 344, 897

Delfosse, X., Forveille, T., Perrier, C., \& Mayor, M. 1998, A\&A, 331, 581

Delfosse, X., Forveille, T., Ségransan, D., Beuzit, J.-L., Udry, S., Perrier, C., \& Mayor, M. 2000, A\&A, 364, 217

Demory, B.-O., et al. 2009, A\&A, in press (arXiv:0906.0602)

Dobler, W., Stix, M., \& Brandenburg, A. 2006, ApJ, 638, 336

Donati, J.-F. 2003, in ASP Conf. Ser. 307, Solar Polarization, ed. J. TrujilloBueno \& J. Sanchez Almeida (San Francisco, CA: ASP), 41

Donati, J.-F., \& Brown, S. F. 1997, A\&A, 326, 1135

Donati, J.-F., Forveille, T., Cameron, A. C., Barnes, J. R., Delfosse, X., Jardine, M. M., \& Valenti, J. A. 2006a, Science, 311, 633

Donati, J.-F., Wade, G. A., Babel, J., Henrichs, H. F., de Jong, J. A., \& Harries, T. J. 2001, MNRAS, 326, 1265

Donati, J.-F., et al. 1997, MNRAS, 291, 658

Donati, J.-F., et al. 2006b, MNRAS, 370, 629

Donati, J.-F., et al. 2008, MNRAS, 390, 545

Epchtein, N. 1997, in The Impact of Large-Scale Near-IR Sky Surveys, ed. F. Garzon, N. Epchtein, A. Omont, B. Burton, \& P. Persi (Dordrecht: Kluwer), 15

ESA 1997, The Hipparcos and Tycho Catalogues (ESA SP-1200; Noordwijk: ESA)

Fleming, T. A. 1998, ApJ, 504, 461

Fleming, T. A., Gioia, I. M., \& Maccacaro, T. 1989, ApJ, 340, 1011

Güdel, M., Schmitt, J. H. M. M., Bookbinder, J. A., \& Fleming, T. A. 1993, ApJ, 415,236

Hallinan, G., Antonova, A., Doyle, J. G., Bourke, S., Lane, C., \& Golden, A. 2008, ApJ, 684, 644

Horne, K. 1986, PASP, 98, 609

Hünsch, M., Schmitt, J. H. M. M., Sterzik, M. F., \& Voges, W. 1999, A\&AS, 135,319

Jardine, M., Barnes, J. R., Donati, J.-F., \& Collier Cameron, A. 1999, MNRAS, 305,35

Johns-Krull, C. M., \& Valenti, J. A. 1996, ApJ, 459, L95

Johns-Krull, C. M., \& Valenti, J. A. 2000, in ASP Conf. Ser. 198, Stellar Clusters and Associations: Convection, Rotation, and Dynamos, ed. R. Pallavicini, G. Micela, \& S. Sciortino (San Francisco, CA: ASP), 371

Johns-Krull, C. M., Valenti, J. A., \& Koresko, C. 1999, ApJ, 516, 900

Küker, M., \& Rüdiger, G. 1997, A\&A, 328, 253
Küker, M., \& Rüdiger, G. 1999, A\&A, 346, 922

Kurucz, R. L. 1993, CDROM \# 13 (ATLAS9 Atmospheric Models) and \#18 (ATLAS9 and SYNTHE Routines, Spectral Line Database; Cambridge; MA: Smithsonian Astrophysical Observatory)

Landolfi, M., \& Landi Degl'Innocenti, E. 1982, Sol. Phys., 78, 355

Leggett, S. K. 1992, ApJS, 82, 531

Leto, G., Pagano, I., Linsky, J. L., Rodonò, M., \& Umana, G. 2000, A\&A, 359, 1035

Liebert, J., Kirkpatrick, J. D., Cruz, K. L., Reid, I. N., Burgasser, A., Tinney, C. G., \& Gizis, J. E. 2003, AJ, 125, 343

Lim, J. 1992, ApJ, 405, L33

Marcy, G. W. 1982, PASP, 94, 989

Martín, E. L., Delfosse, X., Basri, G., Goldman, B., Forveille, T., \& Zapatero Osorio, M. R. 1999, AJ, 118, 2466

Martínez González, M. J., Asensio Ramos, A., Carroll, T. A., Kopf, M., Ramírez Vélez, J. C., \& Semel, M. 2008, A\&A, 486, 637

Mochnacki, S. W., et al. 2002, AJ, 124, 2868

Morales, J. C., Ribas, I., \& Jordi, C. 2008, A\&A, 478, 507

Morin, J., et al. 2008, MNRAS, 390, 567

Mullan, D. J., \& MacDonald, J. 2001, ApJ, 559, 353

Oppenheimer, B. R., Golimowski, D. A., Kulkarni, S. R., Matthews, K., Nakajima, T., Creech-Eakman, M., \& Durrance, S. T. 2001, AJ, 121, 2189

Orlov, V. V., Panchenko, I. E., Rastorguev, A. S., \& Yatsevich, A. V. 1995, AZh, 72,495

Osten, R. A., Phan-Bao, N., Hawley, S. L., Reid, I. N., \& Ojha, R. 2009, ApJ, 700,1750

Parker, E. N. 1975, ApJ, 198, 205

Phan-Bao, N., Martín, E. L., Donati, J.-F., \& Lim, J. 2006, ApJ, 646, L73

Phan-Bao, N., Osten, R. A., Lim, J., Martín, E. L., \& Ho, P. T. P. 2007, ApJ, 658,553

Rao, A. R., \& Singh, K. P. 1990, ApJ, 352, 303

Reid, I. N., Hawley, S. L., \& Gizis, J. E. 1995, AJ, 110, 1838

Reiners, A., \& Basri, G. 2009, A\&A, 496, 787

Ribas, I. 2006, Ap\&SS, 304, 89

Roberts, P. H., \& Stix, M. 1972, A\&A, 18, 453

Robrade, J., \& Schmitt, J. H. M. M. 2009, A\&A, 496, 229

Rüdiger, G., \& Elstner, D. 1994, A\&A, 281, 46

Saar, S. H. 1988, ApJ, 324, 441

Saar, S. H. 1994, in IAU Symp. 154, Infrared Solar Physics, ed. D. M. Rabin et al. (Dordrecht: Kluwer), 493

Schmidt, S. J., Cruz, K. L., Bongiorno, B. J., Liebert, J., \& Reid, I. N. 2007, AJ, 133,2258

Schüssler, M. 1975, A\&A, 38, 263

Ségransan, D., Kervella, P., Forveille, T., \& Queloz, D. 2003, A\&A, 397, L5

Semel, M. 1989, A\&A, 225, 456

Semel, M., Ramírez-Vélez, J. C., Martínez-González, M. J., Asensio Ramos, A., Stift, M. J., López-Ariste, A., \& Leone, F. 2009, A\&A, 504, 1003

Singh, K. P., Drake, S. A., Gotthelf, E. V., \& White, N. E. 1999, ApJ, 512, 874

Skilling, J., \& Bryan, R. K. 1984, MNRAS, 211, 111

Solanki, S. K. 2009, in ASP Conf. Ser. 405 Solar Polarization 5, ed. S. V. Berdyugina, K. N. Nagendra, \& R. Ramelli (San Francisco, CA: ASP), 135

Song, I., Bessell, M., \& Zuckerman, B. 2002, ApJ, 581, L43

Stauffer, J. R., \& Hartmann, L. W. 1986, ApJS, 61, 531

Stelzer, B., Schmitt, J. H. M. M., Micela, G., \& Liefke, C. 2006, A\&A, 460, 35

Tinney, C. G., Mould, J. R., \& Reid, I. N. 1993, AJ, 105, 1045

Vaiana, G. S., et al. 1981, ApJ, 244, 163

Vogt, S. S. 1980, ApJ, 240, 567

Vogt, S. S., Penrod, G. D., \& Hatzes, A. P. 1987, ApJ, 321, 496

Wade, G. A., Donati, J.-F., Landstreet, J. D., \& Shorlin, S. L. S. 2000, MNRAS, 313,851

XMM-SSC 2008, The XMM-Newton 2nd Incremental Source Catalogue (2XMMi Leicester, UK: XMM-SSC)

Young, A., Skumanich, A., \& Harlan, E. 1984, ApJ, 282, 683

Young, A., Skumanich, A., MacGregor, K. B., \& Temple, S. 1990, ApJ, 349, 608

Zuckerman, B., \& Song, I. 2004, ARA\&A, 42, 685 